\title{
\#museuobert: los museos no se detienen ante la pandemia
}

\author{
\#museuobert: museums don't stand still facing the pandemic
}

\author{
Andrea Mejuto ${ }^{\mathrm{a}}$, Irene Gómez ${ }^{\mathrm{b}}$, Carlota Royo $^{\mathrm{c}}$, Enric Zapatero $^{\mathrm{d}}$ \\ a \#museuobert y Nubilum (andrea.mejuto@nubilum.es), b\#museuobert y Coeli Platform \\ (irene.gomez@coeli.cat), ${ }^{\mathrm{c}}$ \#museuobert y Coeli Platform (carlota.royo@coeli.cat). ${ }^{\mathrm{d}}$ \#museuobert y \\ Nubilum (enric.zapatero@nubilum.es).
}

\begin{abstract}
Resumen
Los "confinamientos universales" del sector GLAM (Kużelewska y Tomaszuk, 2020) pusieron de manifiesto la necesidad de reformular la actividad de cada institución para acercarse a la audiencia en el espacio 2.0. En el último año, cuatro de cada cinco museos ha incrementado su presencia digital para llegar a sus públicos, según los últimos informes de Network of European Museums Organisations (NEMO, 2020). El contexto actual, regido por las restricciones sanitarias, ha catalizado la discusión acerca del rol de los museos en el siglo veintiuno.
\end{abstract}

El portal museuobert.cat, puesto en funcionamiento en tan solo dos semanas, es un espacio colectivo de difusión del patrimonio cultural. La iniciativa afloró en abril de 2020 con la firme convicción de estrechar el vínculo entre los museos y las personas en un contexto marcado por el distanciamiento social. Actualmente, el espacio \#museuobert cuenta con un total de veinticuatro museos del territorio catalán y dispone de prácticamente 60.000 piezas en línea. Mediante la creación de una plataforma abierta, plural y centralizada, sumado a la implicación de la comunicación digital, el proyecto \#museuobert se establece como precursor de la cultura colectiva digital para impulsar la difusión de la riqueza patrimonial de ayer, hoy y mañana.

Palabras clave: museos durante la pandemia, colecciones en línea, open GLAM, patrimonio digital, estrategia 2.0.

\footnotetext{
Abstract

The "universal lockdowns" of the GLAM sector (Kużelewska and Tomaszuk, 2020) revealed the need to reformulate the activity of each institution to get closer to the audience through the space 2.0. In the last year, four out of five museums have increased their digital presence to reach their publics, according to the latest reports released by the Network of European Museums
} 
Organizations' (NEMO, 2020). The current context, marked by sanitary restrictions, has catalyzed the discussion concerning the role of museums in the twenty-first century. The museuobert.cat portal, which was set into action in just two weeks, is a collective space for the dissemination of cultural heritage. The initiative emerged in April 2020 with the firm conviction of strengthening the bond between museums and people in a context strictly marked by social distancing. Currently, \#museuobert's scheme counts on the presence of twenty-four museums from the Catalan territory and holds almost 60,000 pieces online. Through the creation of an open, plural and centralised platform, added to the involvement of digital communication, the \#museuobert project establishes itself as a precursor of collective culture online to inspire the dissemination of yesterday's, today's and tomorrow's heritage.

Keywords: museums in lockdown, online collections, open GLAM, digital heritage, strategy 2.0. 


\section{Introducción}

El confinamiento en marzo de 2020 situó a la sociedad en un momento histórico totalmente inédito. El sector cultural fue uno de los más afectados y los museos se encontraron, de la noche a la mañana, obligados a cerrar sus puertas sin una clara previsión para volver. Esta situación generó una laguna comunicativa por parte de los museos, traducido en la necesidad inmediata de difundir sus colecciones digitalmente para hacer frente a los estragos causados por el distanciamiento social y el confinamiento de toda la sociedad en sus casas.

El proyecto \#museuobert llegó para dar voz y visibilidad a todo tipo de museos y entidades, especialmente a aquellos que no disponen de sus colecciones en línea. Gestado en un tiempo récord por las empresas Coeli Platform y Nubilum, que aportan la tecnología y el conocimiento documental respectivamente, el portal de difusión museuobert.cat pretende ayudar a los museos a difundir su patrimonio cultural y a estar presentes en un momento en el que la sociedad debe permanecer en casa.

\section{\#museuobert}

\section{\#museuobert}

Roboto bold

Latterspacingo.osem

Fig. 1 Identidad corporativa

\section{Objetivos}

El lema principal de \#museuobert es "Unimos fuerzas para que los museos no paren" que ilustra, de una forma nítida, el propósito primordial y la voluntad de servicio de esta iniciativa. He aquí los objetivos generales y específicos de \#museuobert como vínculo entre las instituciones culturales y el público general frente a un contexto marcado por el distanciamiento social. 
Tabla 1. Objetivos generales y específicos

\begin{tabular}{|c|c|}
\hline Generales & Específicos \\
\hline $\begin{array}{l}\text { Apoyar a los museos } \\
\text { para que no detengan } \\
\text { su actividad } \\
\text { ante la pandemia }\end{array}$ & $\begin{array}{l}\text { Ofrecer una plataforma tecnológica } \\
\text { y soporte en la sincronización de datos } \\
\text { para difundir el patrimonio cultural } \\
\text { Crear un espacio plural que visibilice } \\
\text { y ponga en valor a todo tipo de museos } \\
\text { Solidificar la presencia en línea de las } \\
\text { instituciones adscritas a la iniciativa } \\
\text { Diseñar un portal user-friendly, } \\
\text { pensado para el visitante virtual } \\
\text { Establecer una conversación bidireccional } \\
\text { a través de canales de comunicación digital } \\
\text { Facilitar la difusión del contenido } \\
\text { por medio de botones de redes sociales } \\
\text { integrados en la página } \\
\text { Consolidar un espacio } 2.0 \text { de } \\
\text { cultura colectiva en línea }\end{array}$ \\
\hline
\end{tabular}

\section{Diseño de la innovación}

Desde un primer momento, el proyecto \#museuobert ha puesto en valor el nexo entre las instituciones culturales y el usuario digital. Partiendo desde este ángulo, los pilares fundamentales de \#museuobert han sido vertebrados a través de las innovaciones detalladas en este apartado.

\subsection{Pasarela horizontal}

La página web se estructura en base a una pasarela horizontal en la cual el usuario puede pasear con total libertad por las obras de los distintos museos sin abandonar el propio entorno 
web. Dicha disposición emerge sobre una single-page, una única página ágil e intuitiva, a modo de galería que proporciona una experiencia fluida y atractiva para el visitante.

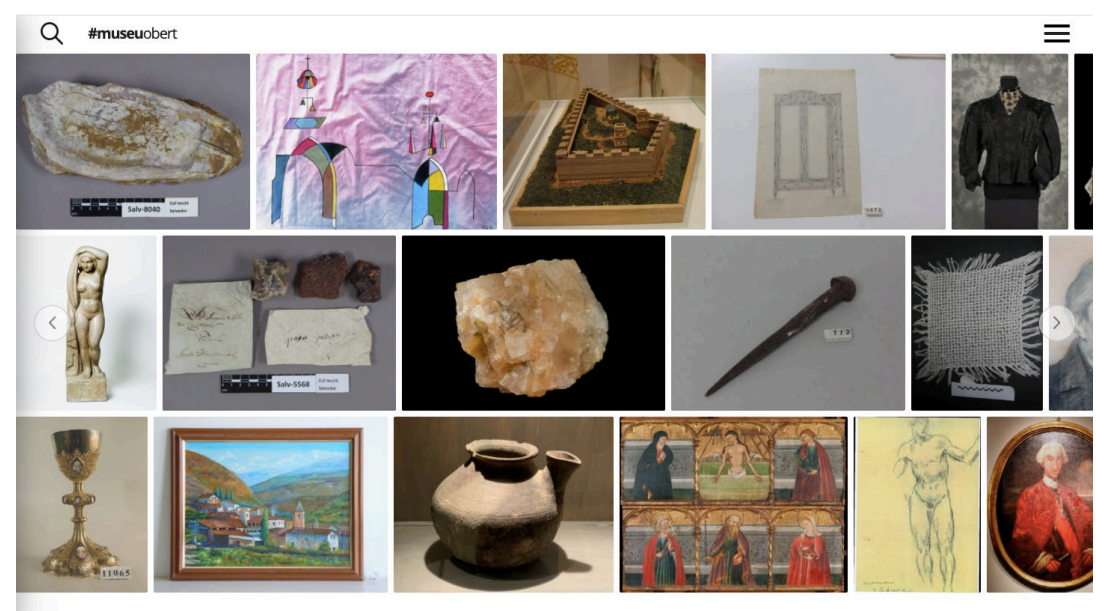

Fig. 2 Disposición de \#museuobert

\subsection{Protagonismo de las piezas}

El epicentro del proyecto lo conforman, en esencia, las piezas de los museos. A través de una presentación minimalista constituida exclusivamente en blanco y negro, el diseño corporativo de \#museuobert ofrece elegancia y nitidez a la hora de otorgar el máximo protagonismo a cada una de las obras. Asimismo, éstas adquieren mayor relevancia gracias a la accesibilidad de la información: bien pasando el cursor sobre una pieza para obtener una breve descripción o bien haciendo clic sobre la misma, donde un zoom in nos proporciona información más extensa sobre una pieza sin abandonar la página en la que nos encontramos.
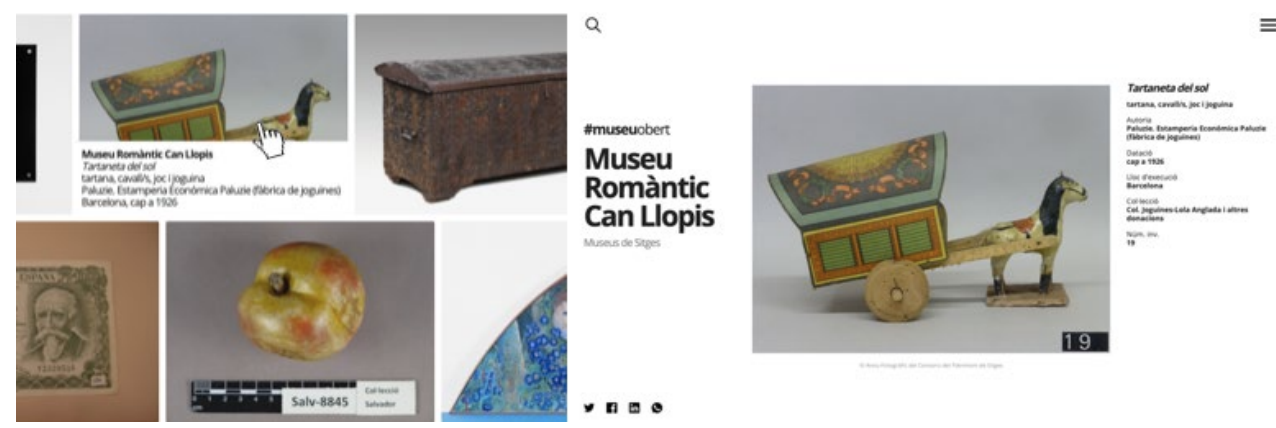

Fig. 3 Accesibilidad de la información 


\subsection{Exposiciones virtuales}

La iniciativa cuenta con una distribución de los contenidos en dos grandes categorías. Por un lado, Museus, donde se muestra el catálogo de museos que forman parte de \#museuobert con acceso a sus colecciones y, por otro lado, Propostes, donde confluyen todas aquellas obras bajo una misma temática y museo. La gran mayoría han sido creadas directamente por cada una de las instituciones culturales, junto a las propuestas genéricas propias de \#museuobert, lo que permite entrelazar discursos entre diversos centros. Los museos autogestionan ellos mismos sus colecciones a través de la plataforma tecnológica cedida por Coeli Platform que, en todo momento, les permite publicar y despublicar sus objetos con total potestad. Actualmente el proyecto cuenta con prácticamente sesenta exposiciones virtuales, que pueden hallarse mediante el siguiente enlace: https://www.museuobert.cat/ca/propostes.

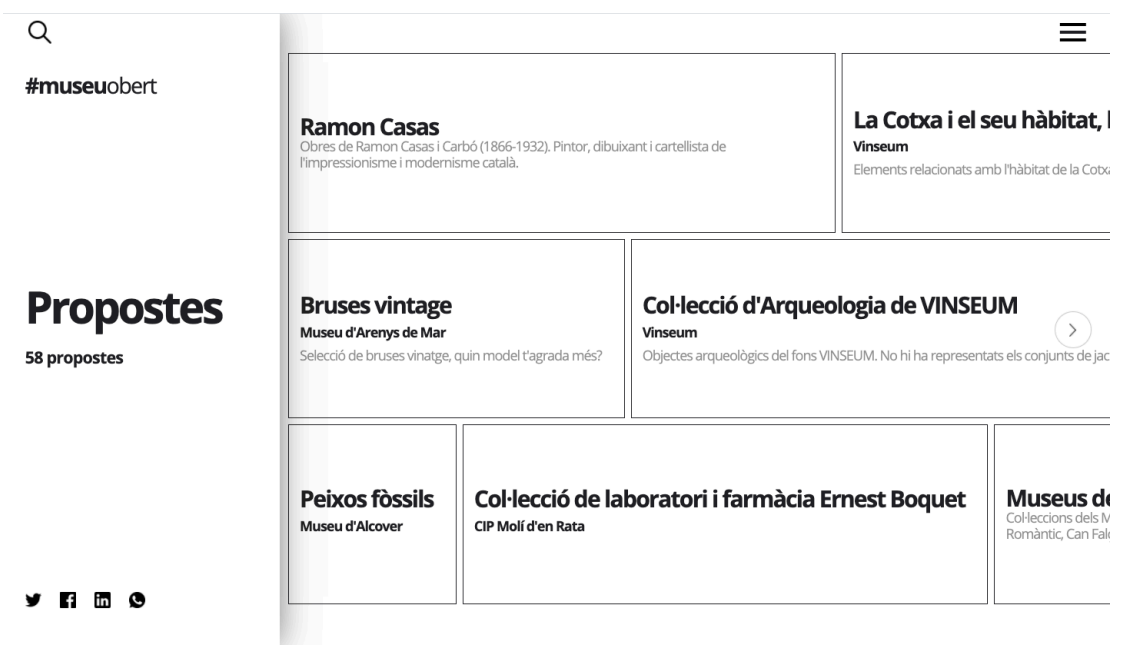

Fig. 4 Pasarela de propuestas expositivas

\subsection{Aleatoriedad}

Una de las particularidades clave del diseño de \#museuobert es la disposición aleatoria del contenido en su home, presentando un orden distinto en cada ocasión. El conjunto de piezas, así como las exposiciones virtuales que hacen aparición, se exhiben entremezcladas -desde retratos y esculturas del siglo XIII hasta minerales tóxicos y juegos de tenedores-, lo que invita al usuario a hacer clic. Al efecto, la variedad de las piezas y la presentación aleatoria se conjugan para proporcionar una experiencia singular, atractiva y sorprendente en cada visita. Si bien la aleatoriedad tiene la ventaja de hacer brotar piezas escondidas, a su vez también puede esconder los objetos de tipologías con mejores porcentajes; para solventar esto, \#museuobert permite que cada institución cultural seleccione hasta 200 piezas 
destacadas para que aparezcan inicialmente, manteniendo así la aleatoriedad global conectada con el resto del conjunto.

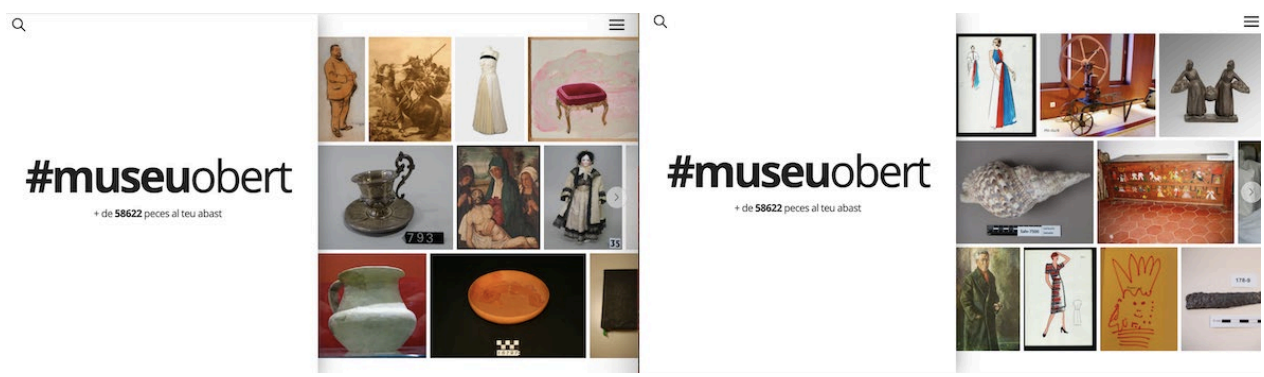

Fig. 5 Cambio de apariencia en cada sesión (diferencia de segundos entre imagen e imagen)

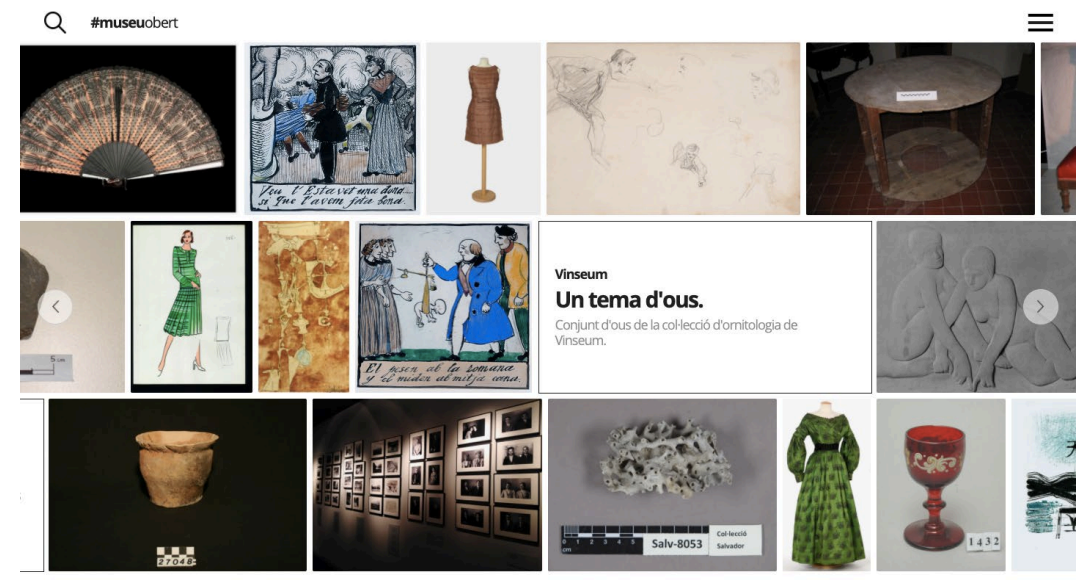

Fig. 6 Propuestas expositivas en la pasarela aleatoria

\subsection{Centralización}

En el territorio, \#museuobert emerge como una página conglomerada precursora de la cultura colectiva en línea. Dada la heterogeneidad de los centros adscritos a este proyecto digital, el equipo de profesionales de Nubilum ha trabajado en la armonización de sinonimias y normalización de nombres con el objetivo de facilitar la recuperación de piezas en el buscador. La centralización, a raíz de esto, constituye uno de los ejes fundamentales de propuestas cuyas identidades son tan plurales y abiertas como la del proyecto \#museuobert. 


\subsection{Interoperabilidad}

La capacidad de integración entre múltiples sistemas de datos y la consecuente variedad de formatos ha sido una tarea clave para el buen desarrollo de la iniciativa. Fundamentalmente, se ha sincronizado toda la información procedente de distintos gestores de colecciones y bases de datos (MuseumPlus, FileMaker, Access, Excel, etc.) y se ha ofrecido el software de Coeli Platform en aquellos casos cuyas colecciones no estaban aún informatizadas. Todo ello, sumado a poder hacer accesible esta iniciativa a cualquier tipo de centro cultural independientemente de que dispongan del inventario informatizado o no, y aproximar las piezas al público gracias a los botones integrados de difusión para los canales sociales, son indudablemente fortalezas destacables del espacio \#museuobert.

\subsection{Integración con redes sociales}

El uso de las redes sociales ha sido clave en la estrategia comunicativa. La creación de un perfil en distintas plataformas ha permitido diversificar los canales en los que se desarrolla la comunicación de \#museuobert. Llegar a distintos usuarios, tanto público en general como museos, adaptándonos a las cualidades de cada plataforma para mostrar las colecciones de los museos más allá de ser una galería de imágenes.

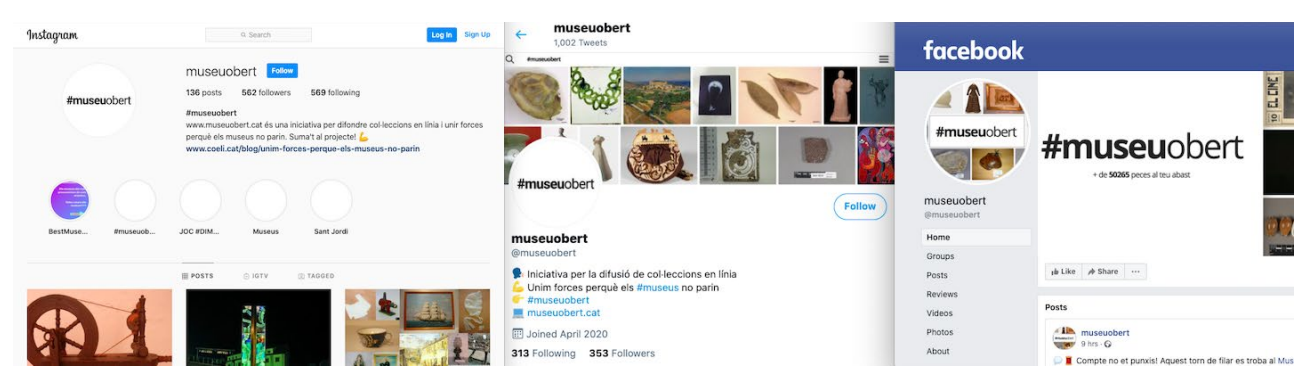

Fig. 7 Perfiles de \#museuobert en las redes

A través de la participación en iniciativas ajenas y propias, mediante el uso de hashtags, se han creado dinámicas y una comunicación directa con el usuario. La estrategia desarrollada para Twitter, Instagram y Facebook, cada una con su particularidad, tienen en común que nos han permitido la integración de las colecciones compartiéndolas tanto desde nuestros perfiles como desde los perfiles de los usuarios a través del propio web. Cada una de las obras dispone de acceso directo a distintos canales (Twitter, Instagram, Facebook y WhatsApp) permitiendo al usuario un flujo comunicativo más directo y sencillo. 


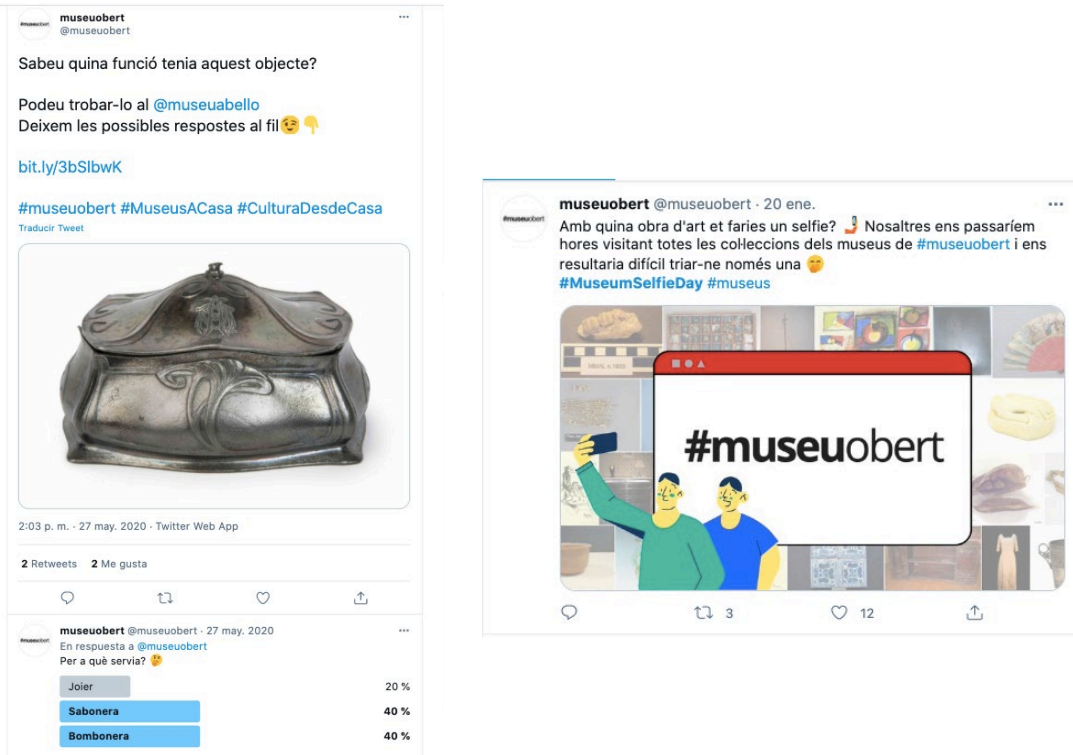

Fig. 7 Ejemplos de dinámicas creadas

(derecha, objetos de las colecciones; izquierda, \#MuseumSelfieDay)

\#museuobert

\section{Museu Pau \\ Casals}

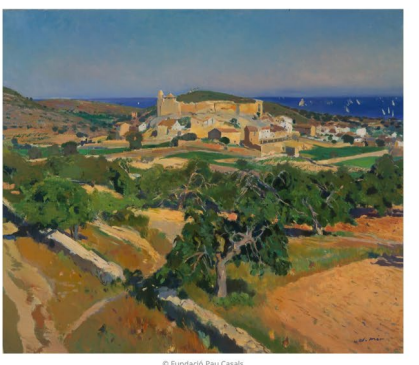

Calafell

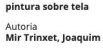

$\substack{\text { Datacio } \\ 1928}^{9}$

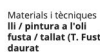

Nüm. de registre
MPC 393

Y If

Fig. 8 Botones integrados para la difusión 


\section{Resultados}

El proyecto \#museuobert salió a la luz a principios de abril de 2020, en pleno estado de alarma. En menos de un año, se han sumado como participantes un total de veinticuatro museos a nivel autonómico y actualmente se recogen cerca de 60.000 piezas publicadas en la web.

Tabla 2. Participantes

(Datos de febrero 2021)

\begin{tabular}{|l|c|}
\hline \multicolumn{1}{|c|}{ Institución cultural } & Número de piezas \\
\hline & \\
Arxiu històric Santa Eulàlia & 705 \\
CIP Molí d'en Rata & 1.135 \\
Espai Cultural dels Canals d'Urgel & 7 \\
Espai Ermengol & 15 \\
Fundació Fita $\quad$ Fundació Stämpfli & 50 \\
Fundació Palau Museu de Maricel $\quad$ Museu del Cau Ferrat $\quad$ Museu Romàntic Can Llopis & 863 \\
Gabinet Salvador $\quad$ Palau de Maricel & 4.630 \\
Herbari de la Universitat de Barcelona & 71 \\
& 1.172 \\
Museus de Can Falç Mar & 131 \\
Sitges $\quad 4.888$ \\
& 4.020 \\
Museu Abelló $\quad 3.716$ \\
Museu de Cervera & 727 \\
Museu de la Vila de Peralada & 865 \\
Museu de les Terres de l'Ebre & 995 \\
Museu d'Alcover & 37 \\
Museu d'Arenys de Mar & 11.376 \\
Museu Municipal de Montcada i Reixac & 2.047 \\
Museu Pau Casals & 6.317 \\
Museu Tèxtil de Terrassa & 540 \\
Vinseum $\quad 442$ \\
\end{tabular}

Durante el transcurso de 2020, el portal web ha recibido más de 50.000 visualizaciones. La amplia variedad de piezas, dada la naturaleza heterogénea del conjunto de participantes, confluye con la deliberada disposición aleatoria para dar lugar a un espacio digital de riqueza patrimonial. 


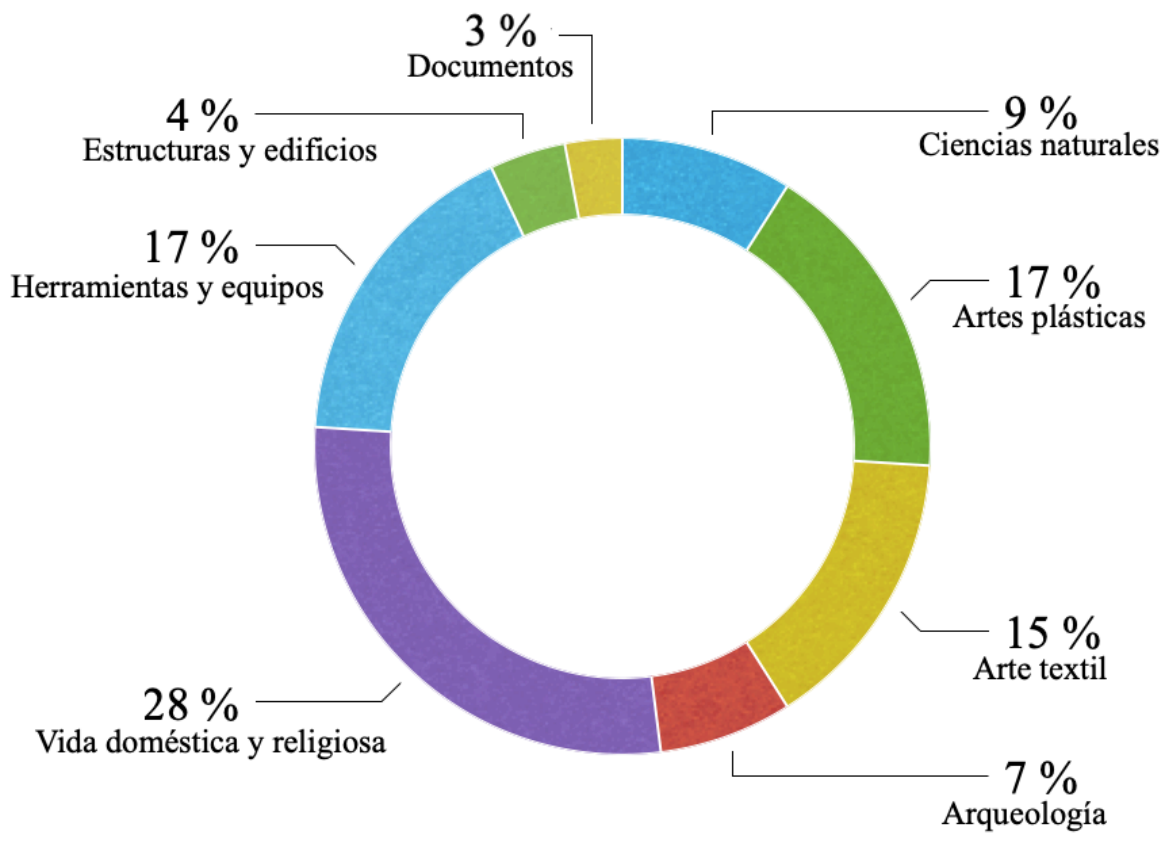

Fig. 9 Clasificación general de las piezas

Sin duda alguna el mayor flujo de usuarios que acceden al web es a través de tráfico directo (35,4\%), es decir, parte de estos usuarios llegan a \#museuobert mediante enlaces que tienen guardados en favoritos del web o a través de enlaces que se encuentran en newsletters, por ejemplo, o mails de otro tipo. Tal y como se ha comentado anteriormente, la comunicación digital ha supuesto una vía vital para la difusión del proyecto \#museuobert ya que constituye un canal democrático de intercambio entre la comunidad del sector GLAM y abre las puertas a nuevas posibilidades narrativas comunitarias (Agostino, Arnaboldi y Lema, 2020). Desde esta óptica, cabe subrayar la importancia de la presencia activa en las redes sociales a través de los perfiles de Facebook (https://www.facebook.com/museuobert), Instagram (https://www.instagram.com/museuobert/) y Twitter (https://twitter.com/museuobert) que recogen alrededor de un 25\% de los usuarios que visitan la página de \#museuobert. En este caso, las redes que más flujo de usuarios atraen son Facebook y Twitter (un total del 93\% del conjunto), destacando sobre todo la primera, sirviendo de puente con la web.

\section{Conclusiones}

El 2020 ha sido, indudablemente, un año que dejará huella en todos los sectores. En el terreno museístico, el paradigma de comunicación digital ha cambiado para siempre; si bien la tendencia ya apuntaba desde hace años hacia estrategias centradas en el contenido propio más allá de la mera difusión de actividades, la pandemia ha acelerado esta transformación. Viéndose forzados a cesar su actividad con la llegada del confinamiento, los museos han tenido que reformular su presencia digital y acercarse a los usuarios poniendo en valor sus 
fondos. El camino de la transformación digital, conectado a un profundo cambio social, permite el acceso a herramientas de comunicación horizontales capaces de estrechar el vínculo entre las instituciones y sus usuarios (Agostino, Arnaboldi y Lema, 2020).

A través de la experiencia del proyecto \#museuobert, se pueden extraer diversos aprendizajes. En primer lugar, convive la necesidad de publicar las colecciones patrimoniales en línea con la escasez de recursos tanto económicos como humanos en el sector cultural. Ante este paisaje, la iniciativa ha cedido gratuitamente los servicios tecnológicos de Coeli Platform y el acompañamiento documental de los profesionales de Nubilum. Otra de las lecciones que ilustra \#museuobert, consecuentemente, es que el propósito del espacio virtual no es el de simular una copia de la realidad sino más bien constituir un entorno por sí mismo. Más allá de las funcionalidades de un catálogo tradicional, los espacios en línea como el de \#museuobert permiten vincular información, crear narrativas cruzadas e interactuar con el público desde distintos canales. Dichas prácticas comunicativas, centradas en un primer momento a raíz de la urgencia inmediata causada por la pandemia, deberán encontrar la forma de afianzarse a largo plazo de una manera sostenible (Ou, 2020).

La cultura digital se ha consolidado como un vector capaz de ofrecer espacios para construir ideas de forma colectiva, demostrando su valor para juntar a las personas en unos tiempos marcados por el distanciamiento social (NEMO, 2020). Los resultados, bajo este foco, demuestran que el proyecto \#museuobert ha sido un actor capaz de apoyar a los museos para solidificar su presencia digital y acercar la cultura a todos los públicos. La buena recepción, tanto por parte de las instituciones como de los usuarios, animan a este proyecto a continuar con su labor de difusión y a seguir creciendo abriéndose paso a nuevos horizontes. Así pues, \#museuobert es y seguirá siendo un museo abierto a las instituciones culturales, a la preservación del patrimonio en el siglo veintiuno y, sobre todo, a las personas. 


\section{Referencias}

AGOSTINO, D., ARNABOLDI, M. y DIAZ LEMA, M. (2020). "New development: COVID-19 as an accelerator of digital transformation in public service delivery". Public Money \& Management, Vol.41 (1), pp. 69-72. <http://dx.doi.org/10.1080/09540962.2020.1764206> [Consulta: 15 de febrero de 2021]

AGOSTINO, D., ARNABOLDI, M. y LAMPIS, A. (2020). "Italian state museums during the COVID19 crisis: from onsite closure to online openness". Museum Management and Curatorship, Vol.35 (4), pp. 362-372. <http://dx.doi.org/10.1080/09647775.2020.1790029> [Consulta: 15 de febrero de 2021]

KUŻELEWSKA, E., TOMASZUK, M. (2020). "European Human Rights Dimension of the Online Access to Cultural Heritage in Times of the COVID-19 Outbreak". Int J Semiot Law $<$ https://doi.org/10.1007/s11196-020-09712-x> [Consulta: 15 de febrero de 2021]

NETWORK OF EUROPEAN MUSEUM (2020). "Survey on the impact of the COVID-19 situation on museums in Europe Final Report" $<$ https://www.nemo.org/fileadmin/Dateien/public/NEMO_documents/NEMO_COVID19_Report_12.05.2020.pdf $>$ [Consulta: 15 de febrero de 2021]

OU, J. (2020) “China Science and Technology Museum boosting fight against COVID-19”. Museum Management and Curatorship, Vol.35 (3), pp. 227-232, $<$ https://doi.org/10.1080/09647775.2020.1762361> [Consulta: 15 de febrero de 2021] 